Supplement of Biogeosciences, 13, 1197-1207, 2016

http://www.biogeosciences.net/13/1197/2016/

doi:10.5194/bg-13-1197-2016-supplement

(C) Author(s) 2016. CC Attribution 3.0 License.

(c) (i)

Supplement of

\title{
Technical Note: Semi-rigid chambers for methane gas flux measurements on tree stems
}

Andy Siegenthaler et al.

Correspondence to: Andy Siegenthaler (mbee777@gmail.com)

The copyright of individual parts of the supplement might differ from the CC-BY 3.0 licence. 
S1. Chamber dimensions and permeabilities $(P)$ determined for all replicates $(n=3)$, calculated from the methane decline slope (Slope), the total chamber volume $\left(V_{\text {tot }}\right)$, the initial concentration gradient between outside and inside $\left(C_{0}-C_{a t m}\right)$ and the gas exchange surface $\left(S_{c}\right) . \mathrm{D}=$ metallic cylinder diameter, $L=$ length, $H=$ height, $T=$ thickness, $C_{0}=$ initial chamber concentration, $C_{a t m}=1.8951$ ppmv, $R^{2}=$ coefficient of

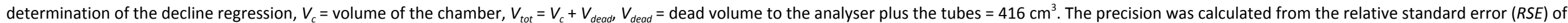
$C_{0}$. The accuracy was determined from the mean of the absolute value difference between $V_{\text {tot }}$ (predicted) and $V_{\text {tot }}^{\prime}$ (observed) devided by $V_{\text {tot }}$

\begin{tabular}{|c|c|c|c|c|c|c|c|c|c|c|c|c|c|c|c|c|c|c|c|c|c|}
\hline \multirow{2}{*}{ Enclosure } & \multirow{2}{*}{ Type } & $D$ & $L$ & $H$ & $T$ & $S_{c}$ & $V_{c}$ & $V_{\text {dead }}$ & $V_{\text {tot }}$ & $t_{0}$ & $t_{1}$ & $t_{2}$ & $C_{0}$ & $C_{1}$ & $C_{2}$ & Slope & $R^{2}$ & $J$ & $P$ & $C_{a t m}$ & $V_{\text {tot }}^{\prime}$ \\
\hline & & $(\mathrm{cm})$ & $(\mathrm{cm})$ & $(\mathrm{cm})$ & $(\mathrm{cm})$ & $(\mathrm{cm} 2)$ & $\left(\mathrm{cm}^{3}\right)$ & $\left(\mathrm{cm}^{3}\right)$ & $\left(\mathrm{cm}^{3}\right)$ & $(\min )$ & $(\min )$ & $(\min )$ & (ppmv) & (ppmv) & (ppmv) & $\left(\mathrm{mg} \mathrm{m}^{-3} \mathrm{~s}^{-1}\right)$ & (n.a.) & $\left(\mathrm{mg} \mathrm{m}^{-2} \mathrm{~s}^{-1}\right)$ & $\left(m^{3} m^{-2} s^{-1}\right)$ & $\mathrm{C}_{\mathrm{atm}}$ & $\left(\mathrm{cm}^{3}\right)$ \\
\hline Semi-rigid & 1-Small sleeve & 15 & 25 & 16 & 1.5 & 330 & 550 & 416 & 966 & 0 & 10 & 20 & 109.1 & 106.4 & 106.1 & $-1.73 E-03$ & 0.804 & $-5.06 \mathrm{E}-05$ & $6.69 \mathrm{E}-07$ & 1.8951 & 933 \\
\hline Semi-rigid & 1-Small sleeve & 15 & 25 & 16 & 1.5 & 330 & 550 & 416 & 966 & 0 & 10 & 20 & 105.7 & 103.4 & 101.6 & $-2.40 \mathrm{E}-03$ & 0.994 & $-7.03 \mathrm{E}-05$ & $9.59 \mathrm{E}-07$ & 1.8951 & 963 \\
\hline Semi-rigid & 1-Small sleeve & 15 & 25 & 16 & 1.5 & 330 & 550 & 416 & 966 & 0 & 10 & 20 & 112.6 & 110.3 & 108.7 & $-2.30 \mathrm{E}-03$ & 0.992 & $-6.73 \mathrm{E}-05$ & $8.61 \mathrm{E}-07$ & 1.8951 & 903 \\
\hline Semi-rigid & 2-Large sleeve & 15 & 30 & 24 & 1.5 & 594 & 990 & 416 & 1406 & 0 & 10 & 20 & 73.5 & 72.3 & 72.0 & $-9.05 E-04$ & 0.914 & $-2.14 \mathrm{E}-05$ & $4.24 \mathrm{E}-07$ & 1.8951 & 1397 \\
\hline Semi-rigid & 2-Large sleeve & 15 & 30 & 24 & 1.5 & 594 & 990 & 416 & 1406 & 0 & 10 & 20 & 71.2 & 70.0 & 69.8 & $-8.36 \mathrm{E}-04$ & 0.858 & $-1.98 \mathrm{E}-05$ & $4.04 \mathrm{E}-07$ & 1.8951 & 1442 \\
\hline Semi-rigid & 2-Large sleeve & 15 & 30 & 24 & 1.5 & 594 & 990 & 416 & 1406 & 0 & 10 & 20 & 69.6 & 68.4 & 67.5 & $-1.22 \mathrm{E}-03$ & 0.993 & $-2.88 \mathrm{E}-05$ & $6.04 \mathrm{E}-07$ & 1.8951 & 1478 \\
\hline Rigid & 3-Acrylic chamber & 15 & 28 & 30 & 6.5 & 1413 & 13165 & 416 & 13581 & 0 & 10 & 20 & 9.3 & 9.2 & 9.1 & $-8.98 \mathrm{E}-05$ & 0.950 & $-8.64 \mathrm{E}-06$ & $1.66 \mathrm{E}-06$ & 1.8951 & 13535 \\
\hline Rigid & 3-Acrylic chamber & 15 & 28 & 30 & 6.5 & 1413 & 13165 & 416 & 13581 & 0 & 10 & 20 & 9.8 & 9.8 & 9.7 & $-6.36 \mathrm{E}-05$ & 0.880 & $-6.11 E-06$ & $1.09 \mathrm{E}-06$ & 1.8951 & 12591 \\
\hline \multirow[t]{5}{*}{ Rigid } & 3-Acrylic chamber & 15 & 28 & 30 & 6.5 & 1413 & 13165 & 416 & 13581 & 0 & 10 & 20 & 9.6 & 9.5 & 9.5 & $-9.31 \mathrm{E}-05$ & 0.963 & $-8.94 \mathrm{E}-06$ & $1.64 \mathrm{E}-06$ & 1.8951 & 12952 \\
\hline & & & & & & & & & & & & & & & & \multicolumn{2}{|c|}{ Enclosure type } & $\begin{array}{c}\begin{array}{c}\text { Precision } \\
(\%)\end{array} \\
\end{array}$ & $\begin{array}{c}\begin{array}{c}\text { Inaccuracy } \\
(\%)\end{array} \\
\end{array}$ & & \\
\hline & & & & & & & & & & & & & & & & \multicolumn{2}{|c|}{ 1-Small sleeve } & 1.82 & 3.39 & & \\
\hline & & & & & & & & & & & & & & & & \multicolumn{2}{|c|}{ 2-Large sleeve } & 1.59 & 2.35 & & \\
\hline & & & & & & & & & & & & & & & & \multicolumn{2}{|c|}{ 3-Acrylic chamber } & 1.68 & 4.09 & & \\
\hline
\end{tabular}


S2. Calculation of the contact distance-to-exchange surface ratio (see text) for the two enclosure types: semi-rigid and rigid; $D=$ stem diameter, $L=$ peripherical length of the chamber or sleeve, $H=$ height of the chamber or sleeve, $T$ $=$ thickness of the chamber or sleeve, Opening = central cylinder diameter, Frame $=$ size of the frame at the edge of the rigid chamber, $V_{c}=$ chamber volume, $E S=$ gas exchange surface, $C D=$ air contact distance of the mobile lines.

\begin{tabular}{lcccccccccc}
\hline Chamber type & $D(\mathrm{~cm})$ & $L(\mathrm{~cm})$ & $H(\mathrm{~cm})$ & $T(\mathrm{~cm})$ & Opening $(\mathrm{cm})$ & Frame $(\mathrm{cm})$ & $V_{c}\left(\mathrm{~cm}^{3}\right)$ & $E S\left(\mathrm{~cm}^{2}\right)$ & $C D(\mathrm{~cm})$ & $C D / E S\left(\mathrm{~m}^{-1}\right)$ \\
\hline Semi-rigid & 15 & 47 & 30 & 1.5 & n.a & n.a & 2120 & 1413 & 154 & 0.109 \\
Rigid cylinder & 15 & 28 & 30 & 6.5 & 18.0 & 5.0 & 13164 & 1413 & 333 & 0.236 \\
\hline
\end{tabular}


S3. Methane concentration changes (ppmv) from tree-stems measured in the manual mode (syringe) as a function of the closure time and species of tree. Full data for plots collected in tropical lowlands of Gigante, Republic of Panama.

\begin{tabular}{|c|c|c|c|}
\hline$\#$ & Run Tree species & Closure time (minutes) & $\mathrm{CH}_{4}$ concentration (ppmv) \\
\hline 1 & 1 Heisteria concinna & 0 & 3.8005 \\
\hline 2 & 1 Heisteria concinna & 5 & 5.0608 \\
\hline 3 & 1 Heisteria concinna & 10 & 6.4977 \\
\hline 4 & 1 Heisteria concinna & 15 & 8.1324 \\
\hline 5 & 2 Heisteria concinna & 0 & 6.0144 \\
\hline 6 & 2 Heisteria concinna & 5 & 20.0046 \\
\hline 7 & 2 Heisteria concinna & 10 & 27.0951 \\
\hline 8 & 2 Heisteria concinna & 15 & 34.0723 \\
\hline 9 & 3 Heisteria concinna & 0 & 3.2066 \\
\hline 10 & 3 Heisteria concinna & 5 & 6.3326 \\
\hline 11 & 3 Heisteria concinna & 10 & 8.9470 \\
\hline 12 & 3 Heisteria concinna & 15 & 9.4321 \\
\hline 13 & 4 Heisteria concinna & 0 & 2.4527 \\
\hline 14 & 4 Heisteria concinna & 5 & 3.6731 \\
\hline 15 & 4 Heisteria concinna & 10 & 5.0027 \\
\hline 16 & 4 Heisteria concinna & 15 & 6.0129 \\
\hline 17 & 5 Heisteria concinna & 0 & 2.9245 \\
\hline 18 & 5 Heisteria concinna & 5 & 6.6789 \\
\hline 19 & 5 Heisteria concinna & 10 & 9.6737 \\
\hline 20 & 5 Heisteria concinna & 15 & 12.0823 \\
\hline 21 & 6 Heisteria concinna & 0 & 5.2829 \\
\hline 22 & 6 Heisteria concinna & 5 & 9.3913 \\
\hline 23 & 6 Heisteria concinna & 10 & 14.1636 \\
\hline 24 & 6 Heisteria concinna & 15 & 21.5350 \\
\hline
\end{tabular}


S4. Methane concentration changes from tree-stems measured in the continuous mode as a function of the closure time and species of tree. Full data for plots collected from the northern boreal zone in Sweden.

\begin{tabular}{|c|c|c|c|}
\hline$\#$ & Run Tree species & Closure time $(\mathrm{s})$ & $\mathrm{CH}_{4}$ concentration (ppmv) \\
\hline 1 & 1 Betula pendula & 0 & 2.0043 \\
\hline 2 & 1 Betula pendula & 3 & 2.0206 \\
\hline 3 & 1 Betula pendula & 6 & 2.0322 \\
\hline 4 & 1 Betula pendula & 9 & 2.0432 \\
\hline 5 & 1 Betula pendula & 12 & 2.0519 \\
\hline 6 & 1 Betula pendula & 15 & 2.0580 \\
\hline 7 & 1 Betula pendula & 18 & 2.0648 \\
\hline 8 & 1 Betula pendula & 21 & 2.0694 \\
\hline 9 & 1 Betula pendula & 24 & 2.0724 \\
\hline 10 & 1 Betula pendula & 27 & 2.0784 \\
\hline 11 & 1 Betula pendula & 30 & 2.0814 \\
\hline 12 & 1 Betula pendula & 33 & 2.0879 \\
\hline 13 & 1 Betula pendula & 36 & 2.0937 \\
\hline 14 & 1 Betula pendula & 39 & 2.0985 \\
\hline 15 & 1 Betula pendula & 42 & 2.0996 \\
\hline 16 & 1 Betula pendula & 45 & 2.1045 \\
\hline 17 & 1 Betula pendula & 48 & 2.1063 \\
\hline 18 & 1 Betula pendula & 51 & 2.1109 \\
\hline 19 & 1 Betula pendula & 54 & 2.1161 \\
\hline 20 & 1 Betula pendula & 57 & 2.1219 \\
\hline 21 & 1 Betula pendula & 60 & 2.1259 \\
\hline 22 & 1 Betula pendula & 63 & 2.1289 \\
\hline 23 & 1 Betula pendula & 66 & 2.1317 \\
\hline 24 & 1 Betula pendula & 69 & 2.1364 \\
\hline 25 & 1 Betula pendula & 72 & 2.1428 \\
\hline 26 & 1 Betula pendula & 75 & 2.1467 \\
\hline 27 & 1 Betula pendula & 78 & 2.1522 \\
\hline 28 & 1 Betula pendula & 81 & 2.1587 \\
\hline 29 & 1 Betula pendula & 84 & 2.1639 \\
\hline 30 & 1 Betula pendula & 87 & 2.1682 \\
\hline 31 & 1 Betula pendula & 90 & 2.1738 \\
\hline 32 & 1 Betula pendula & 93 & 2.1791 \\
\hline 33 & 1 Betula pendula & 96 & 2.1831 \\
\hline 34 & 1 Betula pendula & 99 & 2.1913 \\
\hline 35 & 1 Betula pendula & 102 & 2.1962 \\
\hline 36 & 1 Betula pendula & 105 & 2.2009 \\
\hline 37 & 1 Betula pendula & 108 & 2.2013 \\
\hline 38 & 1 Betula pendula & 111 & 2.2071 \\
\hline 39 & 1 Betula pendula & 114 & 2.2104 \\
\hline 40 & 1 Betula pendula & 117 & 2.2127 \\
\hline 41 & 1 Betula pendula & 120 & 2.2157 \\
\hline
\end{tabular}


S4. Methane concentration changes from tree-stems measured in the continuous mode as a function of the closure time and species of tree. Full data for plots collected from the northern boreal zone in Sweden.

\begin{tabular}{|c|c|c|c|}
\hline$\#$ & Run Tree species & Closure time (s) & $\mathrm{CH}_{4}$ concentration (ppmv) \\
\hline 42 & 1 Betula pendula & 123 & 2.2238 \\
\hline 43 & 1 Betula pendula & 126 & 2.2279 \\
\hline 44 & 1 Betula pendula & 129 & 2.2323 \\
\hline 45 & 1 Betula pendula & 132 & 2.2372 \\
\hline 46 & 1 Betula pendula & 135 & 2.2408 \\
\hline 47 & 1 Betula pendula & 138 & 2.2477 \\
\hline 48 & 1 Betula pendula & 141 & 2.2507 \\
\hline 49 & 1 Betula pendula & 144 & 2.2554 \\
\hline 50 & 1 Betula pendula & 147 & 2.2623 \\
\hline 51 & 1 Betula pendula & 150 & 2.2645 \\
\hline 52 & 1 Betula pendula & 153 & 2.2706 \\
\hline 53 & 1 Betula pendula & 156 & 2.2763 \\
\hline 54 & 1 Betula pendula & 159 & 2.2795 \\
\hline 55 & 1 Betula pendula & 162 & 2.2836 \\
\hline 56 & 1 Betula pendula & 165 & 2.2857 \\
\hline 57 & 1 Betula pendula & 168 & 2.2911 \\
\hline 58 & 1 Betula pendula & 171 & 2.2937 \\
\hline 59 & 1 Betula pendula & 174 & 2.2975 \\
\hline 60 & 1 Betula pendula & 177 & 2.3010 \\
\hline 61 & 1 Betula pendula & 180 & 2.3067 \\
\hline 62 & 1 Betula pendula & 183 & 2.3119 \\
\hline 63 & 1 Betula pendula & 186 & 2.3151 \\
\hline 64 & 1 Betula pendula & 189 & 2.3163 \\
\hline 65 & 1 Betula pendula & 192 & 2.3203 \\
\hline 66 & 1 Betula pendula & 195 & 2.3225 \\
\hline 67 & 1 Betula pendula & 198 & 2.3252 \\
\hline 68 & 1 Betula pendula & 201 & 2.3264 \\
\hline 69 & 1 Betula pendula & 204 & 2.3295 \\
\hline 70 & 1 Betula pendula & 207 & 2.3364 \\
\hline 71 & 1 Betula pendula & 210 & 2.3439 \\
\hline 72 & 1 Betula pendula & 213 & 2.3464 \\
\hline 73 & 1 Betula pendula & 216 & 2.3545 \\
\hline 74 & 1 Betula pendula & 219 & 2.3569 \\
\hline 75 & 1 Betula pendula & 222 & 2.3601 \\
\hline 76 & 1 Betula pendula & 225 & 2.3630 \\
\hline 77 & 1 Betula pendula & 228 & 2.3640 \\
\hline 78 & 1 Betula pendula & 231 & 2.3714 \\
\hline 79 & 1 Betula pendula & 234 & 2.3716 \\
\hline 80 & 1 Betula pendula & 237 & 2.3738 \\
\hline 81 & 1 Betula pendula & 240 & 2.3745 \\
\hline 82 & 1 Betula pendula & 243 & 2.3883 \\
\hline
\end{tabular}


S4. Methane concentration changes from tree-stems measured in the continuous mode as a function of the closure time and species of tree. Full data for plots collected from the northern boreal zone in Sweden.

\begin{tabular}{|c|c|c|c|}
\hline$\#$ & Run Tree species & Closure time (s) & $\mathrm{CH}_{4}$ concentration (ppmv) \\
\hline 83 & 1 Betula pendula & 246 & 2.3813 \\
\hline 84 & 1 Betula pendula & 249 & 2.3872 \\
\hline 85 & 1 Betula pendula & 252 & 2.3926 \\
\hline 86 & 1 Betula pendula & 255 & 2.3944 \\
\hline 87 & 1 Betula pendula & 258 & 2.3957 \\
\hline 88 & 1 Betula pendula & 261 & 2.4004 \\
\hline 89 & 1 Betula pendula & 264 & 2.4060 \\
\hline 90 & 1 Betula pendula & 267 & 2.4100 \\
\hline 91 & 1 Betula pendula & 270 & 2.4136 \\
\hline 92 & 1 Betula pendula & 273 & 2.4169 \\
\hline 93 & 1 Betula pendula & 276 & 2.4197 \\
\hline 94 & 1 Betula pendula & 279 & 2.4220 \\
\hline 95 & 1 Betula pendula & 282 & 2.4247 \\
\hline 96 & 1 Betula pendula & 285 & 2.4252 \\
\hline 97 & 1 Betula pendula & 288 & 2.4283 \\
\hline 98 & 1 Betula pendula & 291 & 2.4289 \\
\hline 99 & 1 Betula pendula & 294 & 2.4308 \\
\hline 100 & 1 Betula pendula & 297 & 2.4331 \\
\hline 101 & 1 Betula pendula & 300 & 2.4342 \\
\hline 102 & 1 Betula pendula & 303 & 2.4386 \\
\hline 103 & 1 Betula pendula & 306 & 2.4427 \\
\hline 104 & 1 Betula pendula & 309 & 2.4467 \\
\hline 105 & 1 Betula pendula & 312 & 2.4527 \\
\hline 106 & 1 Betula pendula & 315 & 2.4564 \\
\hline 107 & 1 Betula pendula & 318 & 2.4603 \\
\hline 108 & 1 Betula pendula & 321 & 2.4641 \\
\hline 109 & 1 Betula pendula & 324 & 2.4671 \\
\hline 110 & 1 Betula pendula & 327 & 2.4695 \\
\hline 111 & 1 Betula pendula & 330 & 2.4746 \\
\hline 112 & 1 Betula pendula & 333 & 2.4799 \\
\hline 113 & 1 Betula pendula & 336 & 2.4842 \\
\hline 114 & 1 Betula pendula & 339 & 2.4906 \\
\hline 115 & 1 Betula pendula & 342 & 2.4952 \\
\hline 116 & 1 Betula pendula & 345 & 2.4970 \\
\hline 117 & 1 Betula pendula & 348 & 2.4980 \\
\hline 118 & 1 Betula pendula & 351 & 2.4980 \\
\hline 119 & 1 Betula pendula & 354 & 2.5004 \\
\hline 120 & 1 Betula pendula & 357 & 2.5015 \\
\hline 121 & 1 Betula pendula & 360 & 2.5001 \\
\hline 122 & 1 Betula pendula & 363 & 2.5003 \\
\hline 123 & 1 Betula pendula & 366 & 2.5030 \\
\hline
\end{tabular}


S4. Methane concentration changes from tree-stems measured in the continuous mode as a function of the closure time and species of tree. Full data for plots collected from the northern boreal zone in Sweden.

\begin{tabular}{|c|c|c|c|}
\hline$\#$ & Run Tree species & Closure time $(\mathrm{s})$ & $\mathrm{CH}_{4}$ concentration (ppmv) \\
\hline 124 & 1 Betula pendula & 369 & 2.5084 \\
\hline 125 & 1 Betula pendula & 372 & 2.5102 \\
\hline 126 & 1 Betula pendula & 375 & 2.5128 \\
\hline 127 & 1 Betula pendula & 378 & 2.5170 \\
\hline 128 & 1 Betula pendula & 381 & 2.5201 \\
\hline 129 & 1 Betula pendula & 384 & 2.5234 \\
\hline 130 & 1 Betula pendula & 387 & 2.5287 \\
\hline 131 & 1 Betula pendula & 390 & 2.5354 \\
\hline 132 & 1 Betula pendula & 393 & 2.5411 \\
\hline 133 & 1 Betula pendula & 396 & 2.5430 \\
\hline 134 & 1 Betula pendula & 399 & 2.5454 \\
\hline 135 & 1 Betula pendula & 402 & 2.5488 \\
\hline 136 & 1 Betula pendula & 405 & 2.5516 \\
\hline 137 & 1 Betula pendula & 408 & 2.5521 \\
\hline 138 & 1 Betula pendula & 411 & 2.5561 \\
\hline 139 & 1 Betula pendula & 414 & 2.5569 \\
\hline 140 & 1 Betula pendula & 417 & 2.5577 \\
\hline 141 & 1 Betula pendula & 420 & 2.5572 \\
\hline 142 & 1 Betula pendula & 423 & 2.5593 \\
\hline 143 & 1 Betula pendula & 426 & 2.5622 \\
\hline 144 & 1 Betula pendula & 429 & 2.5633 \\
\hline 145 & 1 Betula pendula & 432 & 2.5675 \\
\hline 146 & 1 Betula pendula & 435 & 2.5706 \\
\hline 147 & 1 Betula pendula & 438 & 2.5720 \\
\hline 148 & 2 Betula pendula & 0 & 2.1569 \\
\hline 149 & 2 Betula pendula & 3 & 2.1977 \\
\hline 150 & 2 Betula pendula & 6 & 2.2295 \\
\hline 151 & 2 Betula pendula & 9 & 2.2556 \\
\hline 152 & 2 Betula pendula & 12 & 2.2764 \\
\hline 153 & 2 Betula pendula & 15 & 2.2971 \\
\hline 154 & 2 Betula pendula & 18 & 2.3080 \\
\hline 155 & 2 Betula pendula & 21 & 2.3219 \\
\hline 156 & 2 Betula pendula & 24 & 2.3326 \\
\hline 157 & 2 Betula pendula & 27 & 2.3473 \\
\hline 158 & 2 Betula pendula & 30 & 2.3552 \\
\hline 159 & 2 Betula pendula & 33 & 2.3667 \\
\hline 160 & 2 Betula pendula & 36 & 2.3719 \\
\hline 161 & 2 Betula pendula & 39 & 2.3843 \\
\hline 162 & 2 Betula pendula & 42 & 2.3926 \\
\hline 163 & 2 Betula pendula & 45 & 2.3970 \\
\hline 164 & 2 Betula pendula & 48 & 2.4087 \\
\hline
\end{tabular}


S4. Methane concentration changes from tree-stems measured in the continuous mode as a function of the closure time and species of tree. Full data for plots collected from the northern boreal zone in Sweden.

\begin{tabular}{|c|c|c|c|}
\hline$\#$ & Run Tree species & Closure time $(\mathrm{s})$ & $\mathrm{CH}_{4}$ concentration (ppmv) \\
\hline 165 & 2 Betula pendula & 51 & 2.4140 \\
\hline 166 & 2 Betula pendula & 54 & 2.4298 \\
\hline 167 & 2 Betula pendula & 57 & 2.4422 \\
\hline 168 & 2 Betula pendula & 60 & 2.4598 \\
\hline 169 & 2 Betula pendula & 63 & 2.4777 \\
\hline 170 & 2 Betula pendula & 66 & 2.4961 \\
\hline 171 & 2 Betula pendula & 69 & 2.5147 \\
\hline 172 & 2 Betula pendula & 72 & 2.5316 \\
\hline 173 & 2 Betula pendula & 75 & 2.5546 \\
\hline 174 & 2 Betula pendula & 78 & 2.5686 \\
\hline 175 & 2 Betula pendula & 81 & 2.5863 \\
\hline 176 & 2 Betula pendula & 84 & 2.6013 \\
\hline 177 & 2 Betula pendula & 87 & 2.6170 \\
\hline 178 & 2 Betula pendula & 90 & 2.6328 \\
\hline 179 & 2 Betula pendula & 93 & 2.6459 \\
\hline 180 & 2 Betula pendula & 96 & 2.6606 \\
\hline 181 & 2 Betula pendula & 99 & 2.6720 \\
\hline 182 & 2 Betula pendula & 102 & 2.6835 \\
\hline 183 & 2 Betula pendula & 105 & 2.6944 \\
\hline 184 & 2 Betula pendula & 108 & 2.7061 \\
\hline 185 & 2 Betula pendula & 111 & 2.7180 \\
\hline 186 & 2 Betula pendula & 114 & 2.7302 \\
\hline 187 & 2 Betula pendula & 117 & 2.7404 \\
\hline 188 & 2 Betula pendula & 120 & 2.7541 \\
\hline 189 & 2 Betula pendula & 123 & 2.7644 \\
\hline 190 & 2 Betula pendula & 126 & 2.7745 \\
\hline 191 & 2 Betula pendula & 129 & 2.7871 \\
\hline 192 & 2 Betula pendula & 132 & 2.7970 \\
\hline 193 & 2 Betula pendula & 135 & 2.8139 \\
\hline 194 & 2 Betula pendula & 138 & 2.8236 \\
\hline 195 & 2 Betula pendula & 141 & 2.8368 \\
\hline 196 & 2 Betula pendula & 144 & 2.8496 \\
\hline 197 & 2 Betula pendula & 147 & 2.8660 \\
\hline 198 & 2 Betula pendula & 150 & 2.8804 \\
\hline 199 & 2 Betula pendula & 153 & 2.8891 \\
\hline 200 & 2 Betula pendula & 156 & 2.9056 \\
\hline 201 & 2 Betula pendula & 159 & 2.9219 \\
\hline 202 & 2 Betula pendula & 162 & 2.9386 \\
\hline 203 & 2 Betula pendula & 165 & 2.9532 \\
\hline 204 & 2 Betula pendula & 168 & 2.9692 \\
\hline 205 & 2 Betula pendula & 171 & 2.9797 \\
\hline
\end{tabular}


S4. Methane concentration changes from tree-stems measured in the continuous mode as a function of the closure time and species of tree. Full data for plots collected from the northern boreal zone in Sweden.

\begin{tabular}{|c|c|c|c|}
\hline$\#$ & Run Tree species & Closure time $(\mathrm{s})$ & $\mathrm{CH}_{4}$ concentration (ppmv) \\
\hline 206 & 2 Betula pendula & 174 & 2.9927 \\
\hline 207 & 2 Betula pendula & 177 & 3.0096 \\
\hline 208 & 2 Betula pendula & 180 & 3.0177 \\
\hline 209 & 2 Betula pendula & 183 & 3.0313 \\
\hline 210 & 2 Betula pendula & 186 & 3.0409 \\
\hline 211 & 2 Betula pendula & 189 & 3.0541 \\
\hline 212 & 2 Betula pendula & 192 & 3.0669 \\
\hline 213 & 2 Betula pendula & 195 & 3.0779 \\
\hline 214 & 2 Betula pendula & 198 & 3.0916 \\
\hline 215 & 2 Betula pendula & 201 & 3.1065 \\
\hline 216 & 2 Betula pendula & 204 & 3.1189 \\
\hline 217 & 2 Betula pendula & 207 & 3.1305 \\
\hline 218 & 2 Betula pendula & 210 & 3.1428 \\
\hline 219 & 2 Betula pendula & 213 & 3.1539 \\
\hline 220 & 2 Betula pendula & 216 & 3.1673 \\
\hline 221 & 2 Betula pendula & 219 & 3.1824 \\
\hline 222 & 2 Betula pendula & 222 & 3.1956 \\
\hline 223 & 2 Betula pendula & 225 & 3.2076 \\
\hline 224 & 2 Betula pendula & 228 & 3.2210 \\
\hline 225 & 2 Betula pendula & 231 & 3.2344 \\
\hline 226 & 2 Betula pendula & 234 & 3.2492 \\
\hline 227 & 2 Betula pendula & 237 & 3.2614 \\
\hline 228 & 2 Betula pendula & 240 & 3.2753 \\
\hline 229 & 2 Betula pendula & 243 & 3.2858 \\
\hline 230 & 2 Betula pendula & 246 & 3.2989 \\
\hline 231 & 2 Betula pendula & 249 & 3.3147 \\
\hline 232 & 2 Betula pendula & 252 & 3.3251 \\
\hline 233 & 2 Betula pendula & 255 & 3.3369 \\
\hline 234 & 2 Betula pendula & 258 & 3.3474 \\
\hline 235 & 2 Betula pendula & 261 & 3.3620 \\
\hline 236 & 2 Betula pendula & 264 & 3.3765 \\
\hline 237 & 2 Betula pendula & 267 & 3.3866 \\
\hline 238 & 2 Betula pendula & 270 & 3.4017 \\
\hline 239 & 2 Betula pendula & 273 & 3.4117 \\
\hline 240 & 2 Betula pendula & 276 & 3.4222 \\
\hline 241 & 2 Betula pendula & 279 & 3.4349 \\
\hline 242 & 2 Betula pendula & 282 & 3.4463 \\
\hline 243 & 2 Betula pendula & 285 & 3.4576 \\
\hline 244 & 2 Betula pendula & 288 & 3.4724 \\
\hline 245 & 2 Betula pendula & 291 & 3.4836 \\
\hline 246 & 2 Betula pendula & 294 & 3.4972 \\
\hline
\end{tabular}


S4. Methane concentration changes from tree-stems measured in the continuous mode as a function of the closure time and species of tree. Full data for plots collected from the northern boreal zone in Sweden.

\begin{tabular}{|c|c|c|c|}
\hline$\#$ & Run Tree species & Closure time $(\mathrm{s})$ & $\mathrm{CH}_{4}$ concentration (ppmv) \\
\hline 247 & 2 Betula pendula & 297 & 3.5101 \\
\hline 248 & 2 Betula pendula & 300 & 3.5238 \\
\hline 249 & 2 Betula pendula & 303 & 3.5377 \\
\hline 250 & 2 Betula pendula & 306 & 3.5537 \\
\hline 251 & 2 Betula pendula & 309 & 3.5616 \\
\hline 252 & 2 Betula pendula & 312 & 3.5738 \\
\hline 253 & 2 Betula pendula & 315 & 3.5832 \\
\hline 254 & 2 Betula pendula & 318 & 3.5945 \\
\hline 255 & 2 Betula pendula & 321 & 3.6097 \\
\hline 256 & 2 Betula pendula & 324 & 3.6258 \\
\hline 257 & 2 Betula pendula & 327 & 3.6368 \\
\hline 258 & 2 Betula pendula & 330 & 3.6480 \\
\hline 259 & 2 Betula pendula & 333 & 3.6636 \\
\hline 260 & 2 Betula pendula & 336 & 3.6711 \\
\hline 261 & 2 Betula pendula & 339 & 3.6840 \\
\hline 262 & 2 Betula pendula & 342 & 3.6976 \\
\hline 263 & 2 Betula pendula & 345 & 3.7141 \\
\hline 264 & 2 Betula pendula & 348 & 3.7245 \\
\hline 265 & 2 Betula pendula & 351 & 3.7373 \\
\hline 266 & 2 Betula pendula & 354 & 3.7475 \\
\hline 267 & 2 Betula pendula & 357 & 3.7595 \\
\hline 268 & 2 Betula pendula & 360 & 3.7720 \\
\hline 269 & 2 Betula pendula & 363 & 3.7855 \\
\hline 270 & 2 Betula pendula & 366 & 3.8018 \\
\hline 271 & 2 Betula pendula & 369 & 3.8174 \\
\hline 272 & 2 Betula pendula & 372 & 3.8254 \\
\hline 273 & 2 Betula pendula & 375 & 3.8391 \\
\hline 274 & 2 Betula pendula & 378 & 3.8491 \\
\hline 275 & 2 Betula pendula & 381 & 3.8598 \\
\hline 276 & 2 Betula pendula & 384 & 3.8719 \\
\hline 277 & 2 Betula pendula & 387 & 3.8813 \\
\hline 278 & 2 Betula pendula & 390 & 3.8962 \\
\hline 279 & 2 Betula pendula & 393 & 3.9078 \\
\hline 280 & 2 Betula pendula & 396 & 3.9160 \\
\hline 281 & 2 Betula pendula & 399 & 3.9320 \\
\hline 282 & 2 Betula pendula & 402 & 3.9395 \\
\hline 283 & 2 Betula pendula & 405 & 3.9526 \\
\hline 284 & 2 Betula pendula & 408 & 3.9609 \\
\hline 285 & 2 Betula pendula & 411 & 3.9792 \\
\hline 286 & 2 Betula pendula & 414 & 3.9846 \\
\hline 287 & 2 Betula pendula & 417 & 3.9971 \\
\hline
\end{tabular}


S4. Methane concentration changes from tree-stems measured in the continuous mode as a function of the closure time and species of tree. Full data for plots collected from the northern boreal zone in Sweden.

\begin{tabular}{|c|c|c|c|}
\hline$\#$ & Run Tree species & Closure time (s) & $\mathrm{CH}_{4}$ concentration (ppmv) \\
\hline 288 & 2 Betula pendula & 420 & 4.0058 \\
\hline 289 & 2 Betula pendula & 423 & 4.0214 \\
\hline 290 & 2 Betula pendula & 426 & 4.0353 \\
\hline 291 & 2 Betula pendula & 429 & 4.0410 \\
\hline 292 & 3 Leaking Betula pendula & 0 & 1.9360 \\
\hline 293 & 3 Leaking Betula pendula & 2 & 1.9297 \\
\hline 294 & 3 Leaking Betula pendula & 4 & 1.9453 \\
\hline 295 & 3 Leaking Betula pendula & 6 & 1.9589 \\
\hline 296 & 3 Leaking Betula pendula & 8 & 1.9777 \\
\hline 297 & 3 Leaking Betula pendula & 10 & 2.0008 \\
\hline 298 & 3 Leaking Betula pendula & 12 & 2.0214 \\
\hline 299 & 3 Leaking Betula pendula & 14 & 2.0397 \\
\hline 300 & 3 Leaking Betula pendula & 16 & 2.0540 \\
\hline 301 & 3 Leaking Betula pendula & 18 & 2.0654 \\
\hline 302 & 3 Leaking Betula pendula & 20 & 2.0728 \\
\hline 303 & 3 Leaking Betula pendula & 22 & 2.0818 \\
\hline 304 & 3 Leaking Betula pendula & 24 & 2.0884 \\
\hline 305 & 3 Leaking Betula pendula & 26 & 2.0957 \\
\hline 306 & 3 Leaking Betula pendula & 28 & 2.1042 \\
\hline 307 & 3 Leaking Betula pendula & 30 & 2.1104 \\
\hline 308 & 3 Leaking Betula pendula & 32 & 2.1124 \\
\hline 309 & 3 Leaking Betula pendula & 34 & 2.1111 \\
\hline 310 & 3 Leaking Betula pendula & 36 & 2.1135 \\
\hline 311 & 3 Leaking Betula pendula & 38 & 2.1189 \\
\hline 312 & 3 Leaking Betula pendula & 40 & 2.1302 \\
\hline 313 & 3 Leaking Betula pendula & 42 & 2.1406 \\
\hline 314 & 3 Leaking Betula pendula & 44 & 2.1535 \\
\hline 315 & 3 Leaking Betula pendula & 46 & 2.1621 \\
\hline 316 & 3 Leaking Betula pendula & 48 & 2.1777 \\
\hline 317 & 3 Leaking Betula pendula & 50 & 2.2033 \\
\hline 318 & 3 Leaking Betula pendula & 52 & 2.2255 \\
\hline 319 & 3 Leaking Betula pendula & 54 & 2.2456 \\
\hline 320 & 3 Leaking Betula pendula & 56 & 2.2721 \\
\hline 321 & 3 Leaking Betula pendula & 58 & 2.3044 \\
\hline 322 & 3 Leaking Betula pendula & 60 & 2.3370 \\
\hline 323 & 3 Leaking Betula pendula & 62 & 2.3551 \\
\hline 324 & 3 Leaking Betula pendula & 64 & 2.3740 \\
\hline 325 & 3 Leaking Betula pendula & 66 & 2.3882 \\
\hline 326 & 3 Leaking Betula pendula & 68 & 2.4003 \\
\hline 327 & 3 Leaking Betula pendula & 70 & 2.4081 \\
\hline 328 & 3 Leaking Betula pendula & 72 & 2.4157 \\
\hline
\end{tabular}


S4. Methane concentration changes from tree-stems measured in the continuous mode as a function of the closure time and species of tree. Full data for plots collected from the northern boreal zone in Sweden.

\begin{tabular}{|c|c|c|c|}
\hline$\#$ & Run Tree species & Closure time (s) & $\mathrm{CH}_{4}$ concentration (ppmv) \\
\hline 329 & 3 Leaking Betula pendula & 74 & 2.4251 \\
\hline 330 & 3 Leaking Betula pendula & 76 & 2.4303 \\
\hline 331 & 3 Leaking Betula pendula & 78 & 2.4370 \\
\hline 332 & 3 Leaking Betula pendula & 80 & 2.4395 \\
\hline 333 & 3 Leaking Betula pendula & 82 & 2.4389 \\
\hline 334 & 3 Leaking Betula pendula & 84 & 2.4395 \\
\hline 335 & 3 Leaking Betula pendula & 86 & 2.4413 \\
\hline 336 & 3 Leaking Betula pendula & 88 & 2.4453 \\
\hline 337 & 3 Leaking Betula pendula & 90 & 2.4473 \\
\hline 338 & 3 Leaking Betula pendula & 92 & 2.4484 \\
\hline 339 & 3 Leaking Betula pendula & 94 & 2.4466 \\
\hline 340 & 3 Leaking Betula pendula & 96 & 2.4520 \\
\hline 341 & 3 Leaking Betula pendula & 98 & 2.4629 \\
\hline 342 & 3 Leaking Betula pendula & 100 & 2.4768 \\
\hline 343 & 3 Leaking Betula pendula & 102 & 2.4939 \\
\hline 344 & 3 Leaking Betula pendula & 104 & 2.5161 \\
\hline 345 & 3 Leaking Betula pendula & 106 & 2.5398 \\
\hline 346 & 3 Leaking Betula pendula & 108 & 2.5633 \\
\hline 347 & 3 Leaking Betula pendula & 110 & 2.5851 \\
\hline 348 & 3 Leaking Betula pendula & 112 & 2.6028 \\
\hline 349 & 3 Leaking Betula pendula & 114 & 2.6178 \\
\hline 350 & 3 Leaking Betula pendula & 116 & 2.6269 \\
\hline 351 & 3 Leaking Betula pendula & 118 & 2.6334 \\
\hline 352 & 3 Leaking Betula pendula & 120 & 2.6435 \\
\hline 353 & 3 Leaking Betula pendula & 122 & 2.6450 \\
\hline 354 & 3 Leaking Betula pendula & 124 & 2.6407 \\
\hline 355 & 3 Leaking Betula pendula & 126 & 2.6429 \\
\hline 356 & 3 Leaking Betula pendula & 128 & 2.6486 \\
\hline 357 & 3 Leaking Betula pendula & 130 & 2.6486 \\
\hline 358 & 3 Leaking Betula pendula & 132 & 2.6420 \\
\hline 359 & 3 Leaking Betula pendula & 134 & 2.6325 \\
\hline 360 & 3 Leaking Betula pendula & 136 & 2.6355 \\
\hline 361 & 3 Leaking Betula pendula & 138 & 2.6475 \\
\hline 362 & 3 Leaking Betula pendula & 140 & 2.6429 \\
\hline 363 & 3 Leaking Betula pendula & 142 & 2.6335 \\
\hline 364 & 3 Leaking Betula pendula & 144 & 2.6295 \\
\hline 365 & 3 Leaking Betula pendula & 146 & 2.6373 \\
\hline 366 & 3 Leaking Betula pendula & 148 & 2.6404 \\
\hline 367 & 3 Leaking Betula pendula & 150 & 2.6348 \\
\hline 368 & 3 Leaking Betula pendula & 152 & 2.6264 \\
\hline 369 & 3 Leaking Betula pendula & 154 & 2.6215 \\
\hline
\end{tabular}


S4. Methane concentration changes from tree-stems measured in the continuous mode as a function of the closure time and species of tree. Full data for plots collected from the northern boreal zone in Sweden.

\begin{tabular}{|c|c|c|c|}
\hline$\#$ & Run Tree species & Closure time $(\mathrm{s})$ & $\mathrm{CH}_{4}$ concentration (ppmv) \\
\hline 370 & 3 Leaking Betula pendula & 156 & 2.6287 \\
\hline 371 & 3 Leaking Betula pendula & 158 & 2.6456 \\
\hline 372 & 3 Leaking Betula pendula & 160 & 2.6573 \\
\hline 373 & 3 Leaking Betula pendula & 162 & 2.6749 \\
\hline 374 & 3 Leaking Betula pendula & 164 & 2.6879 \\
\hline 375 & 3 Leaking Betula pendula & 166 & 2.6881 \\
\hline 376 & 3 Leaking Betula pendula & 168 & 2.6972 \\
\hline 377 & 3 Leaking Betula pendula & 170 & 2.7054 \\
\hline 378 & 3 Leaking Betula pendula & 172 & 2.7243 \\
\hline 379 & 3 Leaking Betula pendula & 174 & 2.7178 \\
\hline 380 & 3 Leaking Betula pendula & 176 & 2.7146 \\
\hline 381 & 3 Leaking Betula pendula & 178 & 2.6979 \\
\hline 382 & 3 Leaking Betula pendula & 180 & 2.6719 \\
\hline 383 & 3 Leaking Betula pendula & 182 & 2.6480 \\
\hline 384 & 3 Leaking Betula pendula & 184 & 2.6308 \\
\hline 385 & 3 Leaking Betula pendula & 186 & 2.6206 \\
\hline 386 & 3 Leaking Betula pendula & 188 & 2.6214 \\
\hline 387 & 3 Leaking Betula pendula & 190 & 2.6225 \\
\hline 388 & 3 Leaking Betula pendula & 192 & 2.6355 \\
\hline 389 & 3 Leaking Betula pendula & 194 & 2.6411 \\
\hline 390 & 3 Leaking Betula pendula & 196 & 2.6224 \\
\hline 391 & 3 Leaking Betula pendula & 198 & 2.6082 \\
\hline 392 & 3 Leaking Betula pendula & 200 & 2.6075 \\
\hline 393 & 3 Leaking Betula pendula & 202 & 2.6216 \\
\hline 394 & 3 Leaking Betula pendula & 204 & 2.6390 \\
\hline 395 & 3 Leaking Betula pendula & 206 & 2.6500 \\
\hline 396 & 3 Leaking Betula pendula & 208 & 2.6619 \\
\hline 397 & 3 Leaking Betula pendula & 210 & 2.6700 \\
\hline 398 & 3 Leaking Betula pendula & 212 & 2.6773 \\
\hline 399 & 3 Leaking Betula pendula & 214 & 2.6864 \\
\hline 400 & 3 Leaking Betula pendula & 216 & 2.6962 \\
\hline 401 & 3 Leaking Betula pendula & 218 & 2.7031 \\
\hline 402 & 3 Leaking Betula pendula & 220 & 2.7164 \\
\hline 403 & 3 Leaking Betula pendula & 222 & 2.7298 \\
\hline 404 & 3 Leaking Betula pendula & 224 & 2.7411 \\
\hline 405 & 3 Leaking Betula pendula & 226 & 2.7500 \\
\hline 406 & 3 Leaking Betula pendula & 228 & 2.7600 \\
\hline 407 & 3 Leaking Betula pendula & 230 & 2.7703 \\
\hline 408 & 3 Leaking Betula pendula & 232 & 2.7769 \\
\hline 409 & 3 Leaking Betula pendula & 234 & 2.7807 \\
\hline 410 & 3 Leaking Betula pendula & 236 & 2.7906 \\
\hline
\end{tabular}


S4. Methane concentration changes from tree-stems measured in the continuous mode as a function of the closure time and species of tree. Full data for plots collected from the northern boreal zone in Sweden.

\begin{tabular}{|c|c|c|c|}
\hline \# & Run Tree species & Closure time $(\mathrm{s})$ & $\mathrm{CH}_{4}$ concentration (ppmv) \\
\hline 411 & 3 Leaking Betula pendula & 238 & 2.7997 \\
\hline 412 & 3 Leaking Betula pendula & 240 & 2.8076 \\
\hline 413 & 3 Leaking Betula pendula & 242 & 2.8170 \\
\hline 414 & 3 Leaking Betula pendula & 244 & 2.8210 \\
\hline 415 & 3 Leaking Betula pendula & 246 & 2.8194 \\
\hline 416 & 3 Leaking Betula pendula & 248 & 2.8155 \\
\hline 417 & 3 Leaking Betula pendula & 250 & 2.8085 \\
\hline 418 & 3 Leaking Betula pendula & 252 & 2.7968 \\
\hline 419 & 3 Leaking Betula pendula & 254 & 2.7878 \\
\hline 420 & 3 Leaking Betula pendula & 256 & 2.7832 \\
\hline 421 & 3 Leaking Betula pendula & 258 & 2.7863 \\
\hline 422 & 3 Leaking Betula pendula & 260 & 2.7942 \\
\hline 423 & 3 Leaking Betula pendula & 262 & 2.8025 \\
\hline 424 & 3 Leaking Betula pendula & 264 & 2.8204 \\
\hline 425 & 3 Leaking Betula pendula & 266 & 2.8335 \\
\hline 426 & 3 Leaking Betula pendula & 268 & 2.8475 \\
\hline 427 & 3 Leaking Betula pendula & 270 & 2.8535 \\
\hline 428 & 3 Leaking Betula pendula & 272 & 2.8600 \\
\hline 429 & 3 Leaking Betula pendula & 274 & 2.8646 \\
\hline 430 & 3 Leaking Betula pendula & 276 & 2.8743 \\
\hline 431 & 3 Leaking Betula pendula & 278 & 2.8874 \\
\hline 432 & 3 Leaking Betula pendula & 280 & 2.8954 \\
\hline 433 & 3 Leaking Betula pendula & 282 & 2.9044 \\
\hline 434 & 3 Leaking Betula pendula & 284 & 2.9201 \\
\hline 435 & 3 Leaking Betula pendula & 286 & 2.9372 \\
\hline 436 & 3 Leaking Betula pendula & 288 & 2.9812 \\
\hline 437 & 3 Leaking Betula pendula & 290 & 3.0019 \\
\hline 438 & 3 Leaking Betula pendula & 292 & 2.9564 \\
\hline 439 & 3 Leaking Betula pendula & 294 & 2.9113 \\
\hline 440 & 3 Leaking Betula pendula & 296 & 2.8870 \\
\hline 441 & 3 Leaking Betula pendula & 298 & 2.8817 \\
\hline 442 & 3 Leaking Betula pendula & 300 & 2.8857 \\
\hline 443 & 3 Leaking Betula pendula & 302 & 2.8999 \\
\hline 444 & 3 Leaking Betula pendula & 304 & 2.9083 \\
\hline 445 & 3 Leaking Betula pendula & 306 & 2.9153 \\
\hline 446 & 3 Leaking Betula pendula & 308 & 2.9174 \\
\hline 447 & 3 Leaking Betula pendula & 310 & 2.9194 \\
\hline 448 & 3 Leaking Betula pendula & 312 & 2.9212 \\
\hline 449 & 3 Leaking Betula pendula & 314 & 2.9265 \\
\hline 450 & 3 Leaking Betula pendula & 316 & 2.9451 \\
\hline 451 & 3 Leaking Betula pendula & 318 & 2.9598 \\
\hline
\end{tabular}


S4. Methane concentration changes from tree-stems measured in the continuous mode as a function of the closure time and species of tree. Full data for plots collected from the northern boreal zone in Sweden.

\begin{tabular}{|c|c|c|c|}
\hline$\#$ & Run Tree species & Closure time (s) & $\mathrm{CH}_{4}$ concentration (ppmv) \\
\hline 452 & 3 Leaking Betula pendula & 320 & 2.9762 \\
\hline 453 & 3 Leaking Betula pendula & 322 & 2.9953 \\
\hline 454 & 3 Leaking Betula pendula & 324 & 3.0225 \\
\hline 455 & 3 Leaking Betula pendula & 326 & 3.0466 \\
\hline 456 & 3 Leaking Betula pendula & 328 & 3.0618 \\
\hline 457 & 3 Leaking Betula pendula & 330 & 3.0830 \\
\hline 458 & 3 Leaking Betula pendula & 332 & 3.0924 \\
\hline 459 & 3 Leaking Betula pendula & 334 & 3.0939 \\
\hline 460 & 3 Leaking Betula pendula & 336 & 3.0845 \\
\hline 461 & 3 Leaking Betula pendula & 338 & 3.0639 \\
\hline 462 & 3 Leaking Betula pendula & 340 & 3.0486 \\
\hline 463 & 3 Leaking Betula pendula & 342 & 3.0491 \\
\hline 464 & 3 Leaking Betula pendula & 344 & 3.0378 \\
\hline 465 & 3 Leaking Betula pendula & 346 & 3.0244 \\
\hline 466 & 3 Leaking Betula pendula & 348 & 3.0170 \\
\hline 467 & 3 Leaking Betula pendula & 350 & 3.0191 \\
\hline 468 & 3 Leaking Betula pendula & 352 & 3.0283 \\
\hline 469 & 3 Leaking Betula pendula & 354 & 3.0416 \\
\hline 470 & 3 Leaking Betula pendula & 356 & 3.0438 \\
\hline 471 & 3 Leaking Betula pendula & 358 & 3.0467 \\
\hline 472 & 3 Leaking Betula pendula & 360 & 3.0439 \\
\hline 473 & 3 Leaking Betula pendula & 362 & 3.0378 \\
\hline 474 & 3 Leaking Betula pendula & 364 & 3.0339 \\
\hline 475 & 3 Leaking Betula pendula & 366 & 3.0295 \\
\hline 476 & 3 Leaking Betula pendula & 368 & 3.0261 \\
\hline 477 & 3 Leaking Betula pendula & 370 & 3.0274 \\
\hline 478 & 3 Leaking Betula pendula & 372 & 3.0304 \\
\hline 479 & 3 Leaking Betula pendula & 374 & 3.0380 \\
\hline 480 & 3 Leaking Betula pendula & 376 & 3.0479 \\
\hline 481 & 3 Leaking Betula pendula & 378 & 3.0635 \\
\hline 482 & 3 Leaking Betula pendula & 380 & 3.0761 \\
\hline 483 & 3 Leaking Betula pendula & 382 & 3.0800 \\
\hline 484 & 3 Leaking Betula pendula & 384 & 3.0791 \\
\hline 485 & 3 Leaking Betula pendula & 386 & 3.0750 \\
\hline 486 & 3 Leaking Betula pendula & 388 & 3.0683 \\
\hline 487 & 3 Leaking Betula pendula & 390 & 3.0594 \\
\hline 488 & 3 Leaking Betula pendula & 392 & 3.0418 \\
\hline 489 & 3 Leaking Betula pendula & 394 & 3.0287 \\
\hline 490 & 3 Leaking Betula pendula & 396 & 3.0212 \\
\hline 491 & 3 Leaking Betula pendula & 398 & 3.0438 \\
\hline 492 & 3 Leaking Betula pendula & 400 & 3.0688 \\
\hline
\end{tabular}


S4. Methane concentration changes from tree-stems measured in the continuous mode as a function of the closure time and species of tree. Full data for plots collected from the northern boreal zone in Sweden.

\begin{tabular}{|c|c|c|c|}
\hline$\#$ & Run Tree species & Closure time (s) & $\mathrm{CH}_{4}$ concentration (ppmv) \\
\hline 493 & 3 Leaking Betula pendula & 402 & 3.0902 \\
\hline 494 & 3 Leaking Betula pendula & 404 & 3.0489 \\
\hline 495 & 3 Leaking Betula pendula & 406 & 3.0455 \\
\hline 496 & 3 Leaking Betula pendula & 408 & 3.0664 \\
\hline 497 & 3 Leaking Betula pendula & 410 & 3.0882 \\
\hline 498 & 3 Leaking Betula pendula & 412 & 3.0964 \\
\hline 499 & 3 Leaking Betula pendula & 414 & 3.0986 \\
\hline 500 & 3 Leaking Betula pendula & 416 & 3.0939 \\
\hline 501 & 3 Leaking Betula pendula & 418 & 3.0849 \\
\hline 502 & 3 Leaking Betula pendula & 420 & 3.0855 \\
\hline 503 & 3 Leaking Betula pendula & 422 & 3.0903 \\
\hline 504 & 3 Leaking Betula pendula & 424 & 3.0955 \\
\hline 505 & 3 Leaking Betula pendula & 426 & 3.1035 \\
\hline 506 & 3 Leaking Betula pendula & 428 & 3.1134 \\
\hline 507 & 3 Leaking Betula pendula & 430 & 3.1211 \\
\hline 508 & 3 Leaking Betula pendula & 432 & 3.1261 \\
\hline 509 & 3 Leaking Betula pendula & 434 & 3.1231 \\
\hline 510 & 3 Leaking Betula pendula & 436 & 3.1280 \\
\hline 511 & 3 Leaking Betula pendula & 438 & 3.1401 \\
\hline 512 & 3 Leaking Betula pendula & 440 & 3.1601 \\
\hline 513 & 3 Leaking Betula pendula & 442 & 3.1733 \\
\hline 514 & 3 Leaking Betula pendula & 444 & 3.1743 \\
\hline 515 & 3 Leaking Betula pendula & 446 & 3.1700 \\
\hline 516 & 3 Leaking Betula pendula & 448 & 3.1577 \\
\hline 517 & 3 Leaking Betula pendula & 450 & 3.1484 \\
\hline 518 & 4 Pinus sylvestris & 0 & 2.2088 \\
\hline 519 & 4 Pinus sylvestris & 4 & 2.2123 \\
\hline 520 & 4 Pinus sylvestris & 8 & 2.2125 \\
\hline 521 & 4 Pinus sylvestris & 12 & 2.2162 \\
\hline 522 & 4 Pinus sylvestris & 16 & 2.2161 \\
\hline 523 & 4 Pinus sylvestris & 20 & 2.2198 \\
\hline 524 & 4 Pinus sylvestris & 24 & 2.2228 \\
\hline 525 & 4 Pinus sylvestris & 28 & 2.2232 \\
\hline 526 & 4 Pinus sylvestris & 32 & 2.2267 \\
\hline 527 & 4 Pinus sylvestris & 36 & 2.2306 \\
\hline 528 & 4 Pinus sylvestris & 40 & 2.2318 \\
\hline 529 & 4 Pinus sylvestris & 44 & 2.2336 \\
\hline 530 & 4 Pinus sylvestris & 48 & 2.2348 \\
\hline 531 & 4 Pinus sylvestris & 52 & 2.2355 \\
\hline 532 & 4 Pinus sylvestris & 56 & 2.2365 \\
\hline 533 & 4 Pinus sylvestris & 60 & 2.2381 \\
\hline
\end{tabular}


S4. Methane concentration changes from tree-stems measured in the continuous mode as a function of the closure time and species of tree. Full data for plots collected from the northern boreal zone in Sweden.

\begin{tabular}{|c|c|c|c|}
\hline$\#$ & Run Tree species & Closure time $(\mathrm{s})$ & $\mathrm{CH}_{4}$ concentration (ppmv) \\
\hline 534 & 4 Pinus sylvestris & 64 & 2.2387 \\
\hline 535 & 4 Pinus sylvestris & 68 & 2.2402 \\
\hline 536 & 4 Pinus sylvestris & 72 & 2.2404 \\
\hline 537 & 4 Pinus sylvestris & 76 & 2.2429 \\
\hline 538 & 4 Pinus sylvestris & 80 & 2.2453 \\
\hline 539 & 4 Pinus sylvestris & 84 & 2.2456 \\
\hline 540 & 4 Pinus sylvestris & 88 & 2.2458 \\
\hline 541 & 4 Pinus sylvestris & 92 & 2.2464 \\
\hline 542 & 4 Pinus sylvestris & 96 & 2.2466 \\
\hline 543 & 4 Pinus sylvestris & 100 & 2.2506 \\
\hline 544 & 4 Pinus sylvestris & 104 & 2.2517 \\
\hline 545 & 4 Pinus sylvestris & 108 & 2.2527 \\
\hline 546 & 4 Pinus sylvestris & 112 & 2.2557 \\
\hline 547 & 4 Pinus sylvestris & 116 & 2.2562 \\
\hline 548 & 4 Pinus sylvestris & 120 & 2.2589 \\
\hline 549 & 4 Pinus sylvestris & 124 & 2.2615 \\
\hline 550 & 4 Pinus sylvestris & 128 & 2.2626 \\
\hline 551 & 4 Pinus sylvestris & 132 & 2.2632 \\
\hline 552 & 4 Pinus sylvestris & 136 & 2.2656 \\
\hline 553 & 4 Pinus sylvestris & 140 & 2.2665 \\
\hline 554 & 4 Pinus sylvestris & 144 & 2.2705 \\
\hline 555 & 4 Pinus sylvestris & 148 & 2.2713 \\
\hline 556 & 4 Pinus sylvestris & 152 & 2.2726 \\
\hline 557 & 4 Pinus sylvestris & 156 & 2.2741 \\
\hline 558 & 4 Pinus sylvestris & 160 & 2.2744 \\
\hline 559 & 4 Pinus sylvestris & 164 & 2.2758 \\
\hline 560 & 4 Pinus sylvestris & 168 & 2.2791 \\
\hline 561 & 4 Pinus sylvestris & 172 & 2.2791 \\
\hline 562 & 4 Pinus sylvestris & 176 & 2.2806 \\
\hline 563 & 4 Pinus sylvestris & 180 & 2.2824 \\
\hline 564 & 4 Pinus sylvestris & 184 & 2.2822 \\
\hline 565 & 4 Pinus sylvestris & 188 & 2.2836 \\
\hline 566 & 4 Pinus sylvestris & 192 & 2.2861 \\
\hline 567 & 4 Pinus sylvestris & 196 & 2.2878 \\
\hline 568 & 4 Pinus sylvestris & 200 & 2.2899 \\
\hline 569 & 4 Pinus sylvestris & 204 & 2.2909 \\
\hline 570 & 4 Pinus sylvestris & 208 & 2.2933 \\
\hline 571 & 4 Pinus sylvestris & 212 & 2.2937 \\
\hline 572 & 4 Pinus sylvestris & 216 & 2.2933 \\
\hline 573 & 4 Pinus sylvestris & 220 & 2.2967 \\
\hline 574 & 4 Pinus sylvestris & 224 & 2.2981 \\
\hline
\end{tabular}


S4. Methane concentration changes from tree-stems measured in the continuous mode as a function of the closure time and species of tree. Full data for plots collected from the northern boreal zone in Sweden.

\begin{tabular}{|c|c|c|c|}
\hline$\#$ & Run Tree species & Closure time $(\mathrm{s})$ & $\mathrm{CH}_{4}$ concentration (ppmv) \\
\hline 575 & 4 Pinus sylvestris & 228 & 2.2992 \\
\hline 576 & 4 Pinus sylvestris & 232 & 2.3003 \\
\hline 577 & 4 Pinus sylvestris & 236 & 2.3008 \\
\hline 578 & 4 Pinus sylvestris & 240 & 2.3028 \\
\hline 579 & 4 Pinus sylvestris & 244 & 2.3036 \\
\hline 580 & 4 Pinus sylvestris & 248 & 2.3054 \\
\hline 581 & 4 Pinus sylvestris & 252 & 2.3064 \\
\hline 582 & 4 Pinus sylvestris & 256 & 2.3073 \\
\hline 583 & 4 Pinus sylvestris & 260 & 2.3115 \\
\hline 584 & 4 Pinus sylvestris & 264 & 2.3112 \\
\hline 585 & 4 Pinus sylvestris & 268 & 2.3120 \\
\hline 586 & 4 Pinus sylvestris & 272 & 2.3142 \\
\hline 587 & 4 Pinus sylvestris & 276 & 2.3129 \\
\hline 588 & 4 Pinus sylvestris & 280 & 2.3155 \\
\hline 589 & 4 Pinus sylvestris & 284 & 2.3164 \\
\hline 590 & 4 Pinus sylvestris & 288 & 2.3194 \\
\hline 591 & 4 Pinus sylvestris & 292 & 2.3211 \\
\hline 592 & 4 Pinus sylvestris & 296 & 2.3216 \\
\hline 593 & 4 Pinus sylvestris & 300 & 2.3244 \\
\hline 594 & 4 Pinus sylvestris & 304 & 2.3235 \\
\hline 595 & 4 Pinus sylvestris & 308 & 2.3270 \\
\hline 596 & 4 Pinus sylvestris & 312 & 2.3293 \\
\hline 597 & 4 Pinus sylvestris & 316 & 2.3316 \\
\hline 598 & 4 Pinus sylvestris & 320 & 2.3349 \\
\hline 599 & 4 Pinus sylvestris & 324 & 2.3366 \\
\hline 600 & 4 Pinus sylvestris & 328 & 2.3365 \\
\hline 601 & 4 Pinus sylvestris & 332 & 2.3372 \\
\hline 602 & 4 Pinus sylvestris & 336 & 2.3376 \\
\hline 603 & 4 Pinus sylvestris & 340 & 2.3404 \\
\hline 604 & 4 Pinus sylvestris & 344 & 2.3423 \\
\hline 605 & 4 Pinus sylvestris & 348 & 2.3425 \\
\hline 606 & 4 Pinus sylvestris & 352 & 2.3433 \\
\hline 607 & 4 Pinus sylvestris & 356 & 2.3453 \\
\hline 608 & 4 Pinus sylvestris & 360 & 2.3469 \\
\hline 609 & 4 Pinus sylvestris & 364 & 2.3507 \\
\hline 610 & 4 Pinus sylvestris & 368 & 2.3506 \\
\hline 611 & 4 Pinus sylvestris & 372 & 2.3502 \\
\hline 612 & 4 Pinus sylvestris & 376 & 2.3517 \\
\hline 613 & 4 Pinus sylvestris & 380 & 2.3495 \\
\hline 614 & 4 Pinus sylvestris & 384 & 2.3519 \\
\hline 615 & 4 Pinus sylvestris & 388 & 2.3509 \\
\hline
\end{tabular}


S4. Methane concentration changes from tree-stems measured in the continuous mode as a function of the closure time and species of tree. Full data for plots collected from the northern boreal zone in Sweden.

\begin{tabular}{|c|c|c|c|}
\hline$\#$ & Run Tree species & Closure time $(\mathrm{s})$ & $\mathrm{CH}_{4}$ concentration (ppmv) \\
\hline 616 & 4 Pinus sylvestris & 392 & 2.3519 \\
\hline 617 & 4 Pinus sylvestris & 396 & 2.3533 \\
\hline 618 & 4 Pinus sylvestris & 400 & 2.3564 \\
\hline 619 & 4 Pinus sylvestris & 404 & 2.3557 \\
\hline 620 & 4 Pinus sylvestris & 408 & 2.3559 \\
\hline 621 & 4 Pinus sylvestris & 412 & 2.3619 \\
\hline 622 & 4 Pinus sylvestris & 416 & 2.3623 \\
\hline 623 & 4 Pinus sylvestris & 420 & 2.3627 \\
\hline 624 & 5 Betula pendula & 0 & 2.1240 \\
\hline 625 & 5 Betula pendula & 4 & 2.1879 \\
\hline 626 & 5 Betula pendula & 8 & 2.2293 \\
\hline 627 & 5 Betula pendula & 12 & 2.2564 \\
\hline 628 & 5 Betula pendula & 16 & 2.2742 \\
\hline 629 & 5 Betula pendula & 20 & 2.2926 \\
\hline 630 & 5 Betula pendula & 24 & 2.3064 \\
\hline 631 & 5 Betula pendula & 28 & 2.3174 \\
\hline 632 & 5 Betula pendula & 32 & 2.3312 \\
\hline 633 & 5 Betula pendula & 36 & 2.3427 \\
\hline 634 & 5 Betula pendula & 40 & 2.3523 \\
\hline 635 & 5 Betula pendula & 44 & 2.3644 \\
\hline 636 & 5 Betula pendula & 48 & 2.3689 \\
\hline 637 & 5 Betula pendula & 52 & 2.3741 \\
\hline 638 & 5 Betula pendula & 56 & 2.3794 \\
\hline 639 & 5 Betula pendula & 60 & 2.3819 \\
\hline 640 & 5 Betula pendula & 64 & 2.3860 \\
\hline 641 & 5 Betula pendula & 68 & 2.3918 \\
\hline 642 & 5 Betula pendula & 72 & 2.4044 \\
\hline 643 & 5 Betula pendula & 76 & 2.4191 \\
\hline 644 & 5 Betula pendula & 80 & 2.4329 \\
\hline 645 & 5 Betula pendula & 84 & 2.4477 \\
\hline 646 & 5 Betula pendula & 88 & 2.4582 \\
\hline 647 & 5 Betula pendula & 92 & 2.4714 \\
\hline 648 & 5 Betula pendula & 96 & 2.4834 \\
\hline 649 & 5 Betula pendula & 100 & 2.5005 \\
\hline 650 & 5 Betula pendula & 104 & 2.5130 \\
\hline 651 & 5 Betula pendula & 108 & 2.5260 \\
\hline 652 & 5 Betula pendula & 112 & 2.5403 \\
\hline 653 & 5 Betula pendula & 116 & 2.5540 \\
\hline 654 & 5 Betula pendula & 120 & 2.5647 \\
\hline 655 & 5 Betula pendula & 124 & 2.5787 \\
\hline 656 & 5 Betula pendula & 128 & 2.5892 \\
\hline
\end{tabular}


S4. Methane concentration changes from tree-stems measured in the continuous mode as a function of the closure time and species of tree. Full data for plots collected from the northern boreal zone in Sweden.

\begin{tabular}{|c|c|c|c|}
\hline$\#$ & Run Tree species & Closure time $(\mathrm{s})$ & $\mathrm{CH}_{4}$ concentration (ppmv) \\
\hline 657 & 5 Betula pendula & 132 & 2.6012 \\
\hline 658 & 5 Betula pendula & 136 & 2.6110 \\
\hline 659 & 5 Betula pendula & 140 & 2.6228 \\
\hline 660 & 5 Betula pendula & 144 & 2.6363 \\
\hline 661 & 5 Betula pendula & 148 & 2.6490 \\
\hline 662 & 5 Betula pendula & 152 & 2.6580 \\
\hline 663 & 5 Betula pendula & 156 & 2.6681 \\
\hline 664 & 5 Betula pendula & 160 & 2.6792 \\
\hline 665 & 5 Betula pendula & 164 & 2.6922 \\
\hline 666 & 5 Betula pendula & 168 & 2.7051 \\
\hline 667 & 5 Betula pendula & 172 & 2.7172 \\
\hline 668 & 5 Betula pendula & 176 & 2.7300 \\
\hline 669 & 5 Betula pendula & 180 & 2.7385 \\
\hline 670 & 5 Betula pendula & 184 & 2.7501 \\
\hline 671 & 5 Betula pendula & 188 & 2.7616 \\
\hline 672 & 5 Betula pendula & 192 & 2.7748 \\
\hline 673 & 5 Betula pendula & 196 & 2.7870 \\
\hline 674 & 5 Betula pendula & 200 & 2.7970 \\
\hline 675 & 5 Betula pendula & 204 & 2.8076 \\
\hline 676 & 5 Betula pendula & 208 & 2.8219 \\
\hline 677 & 5 Betula pendula & 212 & 2.8308 \\
\hline 678 & 5 Betula pendula & 216 & 2.8423 \\
\hline 679 & 5 Betula pendula & 220 & 2.8526 \\
\hline 680 & 5 Betula pendula & 224 & 2.8650 \\
\hline 681 & 5 Betula pendula & 228 & 2.8757 \\
\hline 682 & 5 Betula pendula & 232 & 2.8866 \\
\hline 683 & 5 Betula pendula & 236 & 2.8978 \\
\hline 684 & 5 Betula pendula & 240 & 2.9111 \\
\hline 685 & 5 Betula pendula & 244 & 2.9198 \\
\hline 686 & 5 Betula pendula & 248 & 2.9340 \\
\hline 687 & 5 Betula pendula & 252 & 2.9504 \\
\hline 688 & 5 Betula pendula & 256 & 2.9497 \\
\hline 689 & 5 Betula pendula & 260 & 2.9693 \\
\hline 690 & 5 Betula pendula & 264 & 2.9803 \\
\hline 691 & 5 Betula pendula & 268 & 2.9920 \\
\hline 692 & 5 Betula pendula & 272 & 3.0032 \\
\hline 693 & 5 Betula pendula & 276 & 3.0125 \\
\hline 694 & 5 Betula pendula & 280 & 3.0232 \\
\hline 695 & 5 Betula pendula & 284 & 3.0363 \\
\hline 696 & 5 Betula pendula & 288 & 3.0491 \\
\hline 697 & 5 Betula pendula & 292 & 3.0558 \\
\hline
\end{tabular}


S4. Methane concentration changes from tree-stems measured in the continuous mode as a function of the closure time and species of tree. Full data for plots collected from the northern boreal zone in Sweden.

\begin{tabular}{|c|c|c|c|}
\hline$\#$ & Run Tree species & Closure time (s) & $\mathrm{CH}_{4}$ concentration (ppmv) \\
\hline 698 & 5 Betula pendula & 296 & 3.0662 \\
\hline 699 & 5 Betula pendula & 300 & 3.0778 \\
\hline 700 & 5 Betula pendula & 304 & 3.0902 \\
\hline 701 & 5 Betula pendula & 308 & 3.1001 \\
\hline 702 & 5 Betula pendula & 312 & 3.1126 \\
\hline 703 & 5 Betula pendula & 316 & 3.1207 \\
\hline 704 & 5 Betula pendula & 320 & 3.1340 \\
\hline 705 & 5 Betula pendula & 324 & 3.1452 \\
\hline 706 & 5 Betula pendula & 328 & 3.1531 \\
\hline 707 & 5 Betula pendula & 332 & 3.1650 \\
\hline 708 & 5 Betula pendula & 336 & 3.1777 \\
\hline 709 & 5 Betula pendula & 340 & 3.1870 \\
\hline 710 & 5 Betula pendula & 344 & 3.2001 \\
\hline 711 & 5 Betula pendula & 348 & 3.2078 \\
\hline 712 & 5 Betula pendula & 352 & 3.2200 \\
\hline 713 & 5 Betula pendula & 356 & 3.2287 \\
\hline 714 & 5 Betula pendula & 360 & 3.2394 \\
\hline 715 & 5 Betula pendula & 364 & 3.2509 \\
\hline 716 & 5 Betula pendula & 368 & 3.2623 \\
\hline 717 & 5 Betula pendula & 372 & 3.2741 \\
\hline 718 & 5 Betula pendula & 376 & 3.2824 \\
\hline 719 & 5 Betula pendula & 380 & 3.2945 \\
\hline 720 & 5 Betula pendula & 384 & 3.3039 \\
\hline 721 & 5 Betula pendula & 388 & 3.3188 \\
\hline 722 & 5 Betula pendula & 392 & 3.3294 \\
\hline 723 & 5 Betula pendula & 396 & 3.3345 \\
\hline 724 & 5 Betula pendula & 400 & 3.3495 \\
\hline 725 & 5 Betula pendula & 404 & 3.3587 \\
\hline 726 & 5 Betula pendula & 408 & 3.3663 \\
\hline 727 & 5 Betula pendula & 412 & 3.3786 \\
\hline 728 & 5 Betula pendula & 416 & 3.3922 \\
\hline 729 & 5 Betula pendula & 420 & 3.3972 \\
\hline 730 & 5 Betula pendula & 424 & 3.4089 \\
\hline 731 & 5 Betula pendula & 428 & 3.4171 \\
\hline 732 & 5 Betula pendula & 432 & 3.4273 \\
\hline 733 & 5 Betula pendula & 436 & 3.4363 \\
\hline 734 & 6 Leaking Pinus sylvestris & 0 & 2.9010 \\
\hline 735 & 6 Leaking Pinus sylvestris & 5 & 2.9142 \\
\hline 736 & 6 Leaking Pinus sylvestris & 8 & 2.9211 \\
\hline 737 & 6 Leaking Pinus sylvestris & 12 & 2.9175 \\
\hline 738 & 6 Leaking Pinus sylvestris & 16 & 2.9134 \\
\hline
\end{tabular}


S4. Methane concentration changes from tree-stems measured in the continuous mode as a function of the closure time and species of tree. Full data for plots collected from the northern boreal zone in Sweden.

\begin{tabular}{|c|c|c|c|}
\hline$\#$ & Run Tree species & Closure time (s) & $\mathrm{CH}_{4}$ concentration (ppmv) \\
\hline 739 & 6 Leaking Pinus sylvestris & 20 & 2.9057 \\
\hline 740 & 6 Leaking Pinus sylvestris & 24 & 2.9022 \\
\hline 741 & 6 Leaking Pinus sylvestris & 28 & 2.9015 \\
\hline 742 & 6 Leaking Pinus sylvestris & 32 & 2.9032 \\
\hline 743 & 6 Leaking Pinus sylvestris & 36 & 2.9074 \\
\hline 744 & 6 Leaking Pinus sylvestris & 40 & 2.9092 \\
\hline 745 & 6 Leaking Pinus sylvestris & 44 & 2.9114 \\
\hline 746 & 6 Leaking Pinus sylvestris & 48 & 2.9114 \\
\hline 747 & 6 Leaking Pinus sylvestris & 52 & 2.9063 \\
\hline 748 & 6 Leaking Pinus sylvestris & 56 & 2.9002 \\
\hline 749 & 6 Leaking Pinus sylvestris & 60 & 2.8964 \\
\hline 750 & 6 Leaking Pinus sylvestris & 64 & 2.8928 \\
\hline 751 & 6 Leaking Pinus sylvestris & 68 & 2.8890 \\
\hline 752 & 6 Leaking Pinus sylvestris & 72 & 2.8912 \\
\hline 753 & 6 Leaking Pinus sylvestris & 76 & 2.8884 \\
\hline 754 & 6 Leaking Pinus sylvestris & 80 & 2.8902 \\
\hline 755 & 6 Leaking Pinus sylvestris & 84 & 2.8893 \\
\hline 756 & 6 Leaking Pinus sylvestris & 88 & 2.8919 \\
\hline 757 & 6 Leaking Pinus sylvestris & 92 & 2.8932 \\
\hline 758 & 6 Leaking Pinus sylvestris & 96 & 2.8939 \\
\hline 759 & 6 Leaking Pinus sylvestris & 100 & 2.8972 \\
\hline 760 & 6 Leaking Pinus sylvestris & 104 & 2.9058 \\
\hline 761 & 6 Leaking Pinus sylvestris & 108 & 2.9151 \\
\hline 762 & 6 Leaking Pinus sylvestris & 112 & 2.9251 \\
\hline 763 & 6 Leaking Pinus sylvestris & 116 & 2.9356 \\
\hline 764 & 6 Leaking Pinus sylvestris & 120 & 2.9483 \\
\hline 765 & 6 Leaking Pinus sylvestris & 124 & 2.9578 \\
\hline 766 & 6 Leaking Pinus sylvestris & 128 & 2.9710 \\
\hline 767 & 6 Leaking Pinus sylvestris & 132 & 2.9814 \\
\hline 768 & 6 Leaking Pinus sylvestris & 136 & 2.9848 \\
\hline 769 & 6 Leaking Pinus sylvestris & 140 & 2.9920 \\
\hline 770 & 6 Leaking Pinus sylvestris & 144 & 2.9946 \\
\hline 771 & 6 Leaking Pinus sylvestris & 148 & 2.9965 \\
\hline 772 & 6 Leaking Pinus sylvestris & 152 & 2.9986 \\
\hline 773 & 6 Leaking Pinus sylvestris & 156 & 2.9986 \\
\hline 774 & 6 Leaking Pinus sylvestris & 160 & 3.0025 \\
\hline 775 & 6 Leaking Pinus sylvestris & 164 & 3.0031 \\
\hline 776 & 6 Leaking Pinus sylvestris & 168 & 3.0066 \\
\hline 777 & 6 Leaking Pinus sylvestris & 172 & 3.0066 \\
\hline 778 & 6 Leaking Pinus sylvestris & 176 & 3.0138 \\
\hline 779 & 6 Leaking Pinus sylvestris & 180 & 3.0216 \\
\hline
\end{tabular}


S4. Methane concentration changes from tree-stems measured in the continuous mode as a function of the closure time and species of tree. Full data for plots collected from the northern boreal zone in Sweden.

\begin{tabular}{|c|c|c|c|}
\hline$\#$ & Run Tree species & Closure time (s) & $\mathrm{CH}_{4}$ concentration (ppmv) \\
\hline 780 & 6 Leaking Pinus sylvestris & 184 & 3.0310 \\
\hline 781 & 6 Leaking Pinus sylvestris & 188 & 3.0467 \\
\hline 782 & 6 Leaking Pinus sylvestris & 192 & 3.0657 \\
\hline 783 & 6 Leaking Pinus sylvestris & 196 & 3.0808 \\
\hline 784 & 6 Leaking Pinus sylvestris & 200 & 3.0962 \\
\hline 785 & 6 Leaking Pinus sylvestris & 204 & 3.1032 \\
\hline 786 & 6 Leaking Pinus sylvestris & 208 & 3.1119 \\
\hline 787 & 6 Leaking Pinus sylvestris & 212 & 3.1187 \\
\hline 788 & 6 Leaking Pinus sylvestris & 216 & 3.1237 \\
\hline 789 & 6 Leaking Pinus sylvestris & 220 & 3.1283 \\
\hline 790 & 6 Leaking Pinus sylvestris & 224 & 3.1255 \\
\hline 791 & 6 Leaking Pinus sylvestris & 228 & 3.1241 \\
\hline 792 & 6 Leaking Pinus sylvestris & 232 & 3.1182 \\
\hline 793 & 6 Leaking Pinus sylvestris & 236 & 3.1070 \\
\hline 794 & 6 Leaking Pinus sylvestris & 240 & 3.0997 \\
\hline 795 & 6 Leaking Pinus sylvestris & 244 & 3.0975 \\
\hline 796 & 6 Leaking Pinus sylvestris & 248 & 3.0977 \\
\hline 797 & 6 Leaking Pinus sylvestris & 252 & 3.1054 \\
\hline 798 & 6 Leaking Pinus sylvestris & 256 & 3.1174 \\
\hline 799 & 6 Leaking Pinus sylvestris & 260 & 3.1194 \\
\hline 800 & 6 Leaking Pinus sylvestris & 264 & 3.1153 \\
\hline 801 & 6 Leaking Pinus sylvestris & 268 & 3.1208 \\
\hline 802 & 6 Leaking Pinus sylvestris & 272 & 3.1225 \\
\hline 803 & 6 Leaking Pinus sylvestris & 276 & 3.1234 \\
\hline 804 & 6 Leaking Pinus sylvestris & 280 & 3.1235 \\
\hline 805 & 6 Leaking Pinus sylvestris & 284 & 3.1282 \\
\hline 806 & 6 Leaking Pinus sylvestris & 288 & 3.1304 \\
\hline 807 & 6 Leaking Pinus sylvestris & 292 & 3.1345 \\
\hline 808 & 6 Leaking Pinus sylvestris & 296 & 3.1329 \\
\hline 809 & 6 Leaking Pinus sylvestris & 300 & 3.1345 \\
\hline 810 & 6 Leaking Pinus sylvestris & 304 & 3.1402 \\
\hline 811 & 6 Leaking Pinus sylvestris & 308 & 3.1467 \\
\hline 812 & 6 Leaking Pinus sylvestris & 312 & 3.1527 \\
\hline 813 & 6 Leaking Pinus sylvestris & 316 & 3.1605 \\
\hline 814 & 6 Leaking Pinus sylvestris & 320 & 3.1659 \\
\hline 815 & 6 Leaking Pinus sylvestris & 324 & 3.1755 \\
\hline 816 & 6 Leaking Pinus sylvestris & 328 & 3.1844 \\
\hline 817 & 6 Leaking Pinus sylvestris & 332 & 3.1981 \\
\hline 818 & 6 Leaking Pinus sylvestris & 336 & 3.2175 \\
\hline 819 & 6 Leaking Pinus sylvestris & 340 & 3.2432 \\
\hline 820 & 6 Leaking Pinus sylvestris & 344 & 3.2651 \\
\hline
\end{tabular}


S4. Methane concentration changes from tree-stems measured in the continuous mode as a function of the closure time and species of tree. Full data for plots collected from the northern boreal zone in Sweden.

\begin{tabular}{|c|c|c|c|}
\hline$\#$ & Run Tree species & Closure time (s) & $\mathrm{CH}_{4}$ concentration (ppmv) \\
\hline 821 & 6 Leaking Pinus sylvestris & 348 & 3.2816 \\
\hline 822 & 6 Leaking Pinus sylvestris & 352 & 3.2887 \\
\hline 823 & 6 Leaking Pinus sylvestris & 356 & 3.2940 \\
\hline 824 & 6 Leaking Pinus sylvestris & 360 & 3.3014 \\
\hline 825 & 6 Leaking Pinus sylvestris & 364 & 3.3086 \\
\hline 826 & 6 Leaking Pinus sylvestris & 368 & 3.3189 \\
\hline 827 & 6 Leaking Pinus sylvestris & 372 & 3.3282 \\
\hline 828 & 6 Leaking Pinus sylvestris & 376 & 3.3390 \\
\hline 829 & 6 Leaking Pinus sylvestris & 380 & 3.3501 \\
\hline 830 & 6 Leaking Pinus sylvestris & 384 & 3.3536 \\
\hline 831 & 6 Leaking Pinus sylvestris & 388 & 3.3630 \\
\hline 832 & 6 Leaking Pinus sylvestris & 392 & 3.3668 \\
\hline 833 & 6 Leaking Pinus sylvestris & 396 & 3.3703 \\
\hline 834 & 6 Leaking Pinus sylvestris & 400 & 3.3759 \\
\hline 835 & 6 Leaking Pinus sylvestris & 404 & 3.3802 \\
\hline 836 & 6 Leaking Pinus sylvestris & 408 & 3.3799 \\
\hline 837 & 6 Leaking Pinus sylvestris & 412 & 3.3862 \\
\hline 838 & 6 Leaking Pinus sylvestris & 416 & 3.3860 \\
\hline 839 & 6 Leaking Pinus sylvestris & 420 & 3.3907 \\
\hline 840 & 6 Leaking Pinus sylvestris & 424 & 3.3975 \\
\hline
\end{tabular}

